\title{
Engineering the Collapse of Lifetime Distribution of Nitrogen-Vacancy Centers in Nanodiamonds
}

\author{
H. Li ${ }^{1, *}$, J. Y. Ou ${ }^{1}$, B. Gholipour ${ }^{1}$, J. K. So ${ }^{2}$, D. Piccinotti ${ }^{1}$, V. A. Fedotov ${ }^{1}$, N. Papasimakis ${ }^{1}$ \\ ${ }^{1}$ Optoelectronics Research Center \& Center for Photonic Metamaterials, University of \\ Southampton, Southampton SO17 1BJ, UK \\ ${ }^{2}$ Center for Disruptive Photonic Technologies, School of Physical and Mathematical \\ Sciences and The Photonics Institute, Nanyang Technological University, Singapore \\ 637371, Singapore \\ *H.Li@soton.ac.uk
}

\begin{abstract}
We demonstrate experimentally that the distribution of the decay rates of nitrogen-vacancy centers in diamond becomes narrower by over five times for nanodiamonds embedded in thin chalcogenide films.
\end{abstract}

Keywords: diamond NV centers, cathodoluminescence, spontaneous emission rate, lifetime, chalcogenide

The nitrogen-vacancy (NV) center is a point defect in diamond formed by a lattice vacancy paired with a nitrogen atom, which replaces the carbon atom adjacent to the vacancy. ${ }^{1} \mathrm{NV}$ centers are fluorescent in the red part of the optical spectrum and, individually, can act as welllocalized sources of single photons with high photostability even at room temperature. ${ }^{2-5}$ Two charge states are known for $\mathrm{NV}$ centers, namely $\mathrm{NV}^{0}$ and $\mathrm{NV}^{-1}{ }^{1}$ They exhibit zero phonon lines (ZPLs) centered at $575 \mathrm{~nm}$ and $637 \mathrm{~nm}$, respectively, with characteristic broad phonon sidebands at room temperature. ${ }^{1}$ There are also two distinct lifetimes for $\mathrm{NV}^{0}$ and $\mathrm{NV}^{-}$centres. ${ }^{6}$ Typically only emission from the $\mathrm{NV}^{0}$ state is observed in cathodoluminescence experiments at room temperature. ${ }^{7-16}$ The intensity and spectrum of NV emission are highly sensitive to external perturbations, such as temperature, strain, electric and magnetic fields. ${ }^{1,9}$ Moreover, subwavelength confinement of optical fields in metallic nanostructures can strongly modify the emission decay rates of the NV centres when coupled to surface plasmon resonance. ${ }^{17-20}$ Although naturally present in large single-crystal diamonds, NV centers have been routinely produced in nanocrystals (nanodiamonds), which allow efficient extraction of NV fluorescence and enable integration with optical microresonators, thus holding promise as an accessible and cost efficient optical quantum platform for sensing, communication and computing. ${ }^{21-26} \mathrm{NV}$ centers in nanodiamonds (NDs) can also be used as scanning-probes for fluorescence lifetime imaging microscopy, ${ }^{27}$ especially given their good bio-compatibility. ${ }^{28}$ Yet, real-life applications of NV centers in NDs has been difficult in view of the broad distribution of their lifetimes. The broadening results from strong variations of both radiative and nonradiative decay rates, and quantum efficiency of NV centers, ${ }^{29}$ which in practice are controlled by many factors, such as crystal strain, ${ }^{29}$ contamination during fabrication, ${ }^{30}$ coupling to Mie resonance of NDs, ${ }^{31,32}$ locations and orientations of NV centers within NDs, ${ }^{33,34}$ and the presence of large ensembles of NV centers in NDs. ${ }^{12}$

In this Letter, we report that the statistics of the decay rates of NV centers can be efficiently controlled by changing the immediate dielectric environment of NDs. We demonstrate this 
experimentally by characterising the lifetime distribution for large ensembles of NV centers in NDs embedded in thin chalcogenide films, the optical constants of which can be tuned continuously from plasmonic to lossy dielectric. Our analysis indicates that the distribution of lifetimes can become narrower by over five times, featuring a spread of $1 \mathrm{~ns}$ around the average lifetime of $11 \mathrm{~ns}$.

The NDs in our study had an average size of $120 \mathrm{~nm}$ and contained $\sim 1200 \mathrm{NV}^{0}$ (neutral) centers each (obtained from Sigma Aldrich). As a host for NDs we employed a binary alloy of antimony telluride (SbTe), which is an archetypal chalcogenide. NDs were embedded in SbTe using the following procedure. First, a thin film of SbTe of varying composition and ranging in thickness, $d / 2$, from 20 to $50 \mathrm{~nm}$ was deposited over a $28 \mathrm{~mm} \times 28 \mathrm{~mm}$ large area of silicon ( $\mathrm{Si}$ ) substrate using a high-throughput physical vapour deposition system. It was equipped with off-axis Knudsen cell sources symmetrically arranged around the target substrate, which was held under $\leq 10^{-8}$ mbar vacuum at room temperature. The density gradient of $\mathrm{Sb}$ and $\mathrm{Te}$ (each of $\geq 99.9999 \%$ purity) along the substrate was independently controlled using fixed wedge shutters, which ensured that for every SbTe composition (i.e., at every point on the substrate) the atomic components mix simultaneously. ${ }^{35}$ During the synthesis of the film, the phase of the deposited SbTe alloy was formed directly as amorphous. ${ }^{36}$ The obtained SbTe film was then coated via drop casting with a dispersion of NDs, which was prepared by diluting NDs in methanol and mixing the solution for $10 \mathrm{~min}$ in an ultrasonic bath. After methanol evaporated, a second SbTe film (with thickness $d / 2$ ) was deposited over the first SbTe film using the same procedure as above, which yielded an SbTe film with overall thickness $d$ incorporating NDs. Figure 1(a) shows a fragment of the sample featuring ND clusters covered by SbTe. As a reference, we also prepared a sample containing exposed NDs, where the dispersion of NDs was applied onto an SbTe film of thickness $d$, i.e. after the second round of the deposition. All samples were vacuum sealed immediately upon production, and also between characterisation measurements.

The complex permittivity of the synthesized SbTe films was a strong function of composition. It was measured using a variable angle spectroscopic ellipsometer (J. A. Woollam M2000) with an automated stage programmed to sample a $10 \times 10$ point array within the central $19 \mathrm{~mm} \times 19 \mathrm{~mm}$ region (point-to-point separation $2.1 \mathrm{~mm}$ ). Figures 1(b) and 1(c) show the real, $\operatorname{Re} \varepsilon$, and imaginary, $\operatorname{Im} \varepsilon$, parts of SbTe permittivity mapped across the samples at a wavelength of $575 \mathrm{~nm}$ (which corresponds to the zero phonon line of $\mathrm{NV}^{0}$ centers). Re $\varepsilon$ is seen to vary from -23 to 13 crossing zero for a wide range of compositions. Im $\varepsilon$ remains fairly high for all compositions, in the range 15 - 30. Variation in the thickness of the obtained SbTe films is also inherent to the process of synthesizing films from two off-axis sources with wedge shutters. ${ }^{36,37}$ The thickness was measured using a stylus profilometer along the external edges of the film and then interpolated across the central area. Figure 1(d) reveals that the thickness of SbTe films, $d$, obtained after two rounds of deposition varied across the samples from about 40 to $100 \mathrm{~nm}$. 


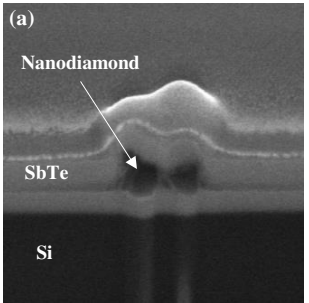

(c) $\operatorname{Im} \varepsilon$ of SbTe film at $\lambda=575 \mathrm{~nm}$

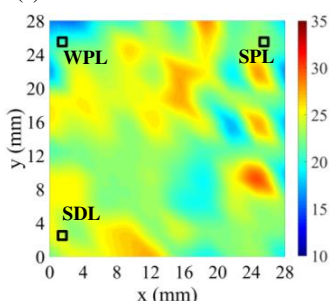

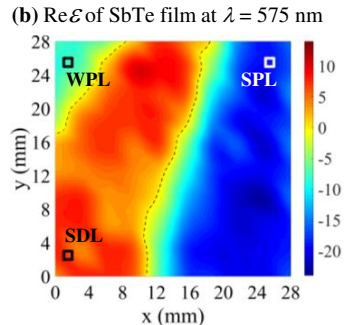

(d) Thickness of SbTe film (nm)

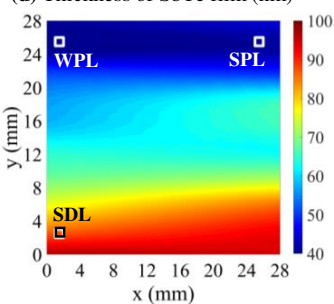

Figure 1. (a) SEM image of nanodiamonds embedded in SbTe films. The scale bar is $200 \mathrm{~nm}$. (b) \& (c) Real and imaginary parts of the permittivity of SbTe films mapped at the wavelength of $575 \mathrm{~nm}$. Dashed curves in (b) trace $\operatorname{Re} \varepsilon=0$. (d) Interpolated spatial map of the thickness of SbTe film, $d$, after two rounds of deposition. Squares in (b-d) mark the areas of the samples where the lifetime statistics of nitrogen-vacancy centers were collected: WPL - weakly plasmonic, SPL - strongly plasmonic, SDL - strongly dielectric.

The lifetimes of $\mathrm{NV}^{0}$ centers were characterized using time-resolved cathodoluminescence (TR-CL). The measurements were performed at room temperature with a scanning electron microscope (SEM) operating at $10 \mathrm{kV}$ in fixed-spot mode. An SEM beam blanker was driven with a wave function generator, which produced pulsed modulation of an electron beam and ensured the synchronization required for implementing time-correlated single-photon counting. In each TR-CL measurement, the beam current was maintained in the range $1.7-1.9 \mathrm{nA}$. The light emitted by the sample via cathodoluminescence was collected and collimated by a parabolic mirror. The collected light was then directed to the entrance slit of a VIS/NIR spectrometer Horiba iHR320, which allowed selecting photons in a $3.6 \mathrm{~nm}$ wide spectral window centered at the $\mathrm{ZPL}$ of $\mathrm{NV}^{0}$. The filtered photons were registered with a single photon detector and the photon counts were arranged into time-correlated histograms (see figure 2(a)). The lifetimes of $\mathrm{NV}^{0}$ centers were extracted by fitting the histograms with a bi-exponential function

$$
I(t)=a_{1}\left(1-\delta\left(t+a_{2}\right)\right)+0.5 a_{1} \delta\left(t+a_{2}\right)\left(e^{-\frac{t+a_{2}}{\tau_{1}}}+e^{-\frac{t+a_{2}}{\tau_{2}}}\right)+a_{3},
$$

where the fast exponential term corresponds to the carrier lifetime, ${ }^{38}$ while the slow exponential term represents the decay rate of $\mathrm{NV}^{0}$ centers.

The lifetime of $\mathrm{NV}^{0}$ centers was characterized in three distinct areas of SbTe films, which are marked in figures 1(b)-1(d) by squares. They all had similar imaginary parts of the permittivity (at the level of $\sim 20$ ) and exhibited strong plasmonic response (SPL) with $\operatorname{Re} \varepsilon=-15$, weak plasmonic response (WPL) with $\operatorname{Re} \varepsilon=-7$ and strong dielectric response (SDL) with $\operatorname{Re} \varepsilon=$ 7 , respectively. For each area, we selected a total of $15 \mathrm{ND}$ clusters. The results of our measurements for $\mathrm{NV}^{0}$ lifetimes are summarized in figure 2(b) and for the carrier lifetimes in 
Supplementary Material. Evidently, the reference sample featuring NDs on top of SbTe film displays a large variation of the lifetimes for all three areas with the spread exceeding $7 \mathrm{~ns}$ and the average lifetime of $\mathrm{NV}^{0}$ centers nearing $20 \mathrm{~ns}$, which is consistent with values reported in the literature. ${ }^{12}$ At the same time, the average lifetimes of $\mathrm{NV}^{0}$ centers in NDs incorporated by SbTe film are seen to become a factor of two shorter, converging at $11 \mathrm{~ns}$ for all three areas. More intriguingly, the spread of the lifetimes for this sample is reduced dramatically and becomes $\sim 1 \mathrm{~ns}$ in the SPL and WPL areas, and $\sim 2 \mathrm{~ns}$ in the SDL area. Similar variations are observed in figure 2 (c) for the distributions of the brightness of $\mathrm{NV}^{0}$ centers, which we also characterised during TR-CL measurements. More specifically, the brightness data exhibit a wide spread for NDs placed on top of an SbTe film regardless of the local dielectric response of the film, but the spread becomes narrower by at least a factor of three for NDs incorporated into the SbTe film. The average brightness of $\mathrm{NV}^{0}$ centers appears to also decrease, by about five times, for NDs embedded in the SbTe film, following the decrease of their average lifetime as revealed by figure 2(b). We note that any variations in the conformality of the deposited SbTe film do not affect appreciably lifetime statistics of embedded NDs. Indeed, such variations would result in different thickness of SbTe shell around NDs but, as evident from figures 1(d) and 2(b), the average and spread of lifetime distributions depend very little on the thickness of SbTe film covering NDs.
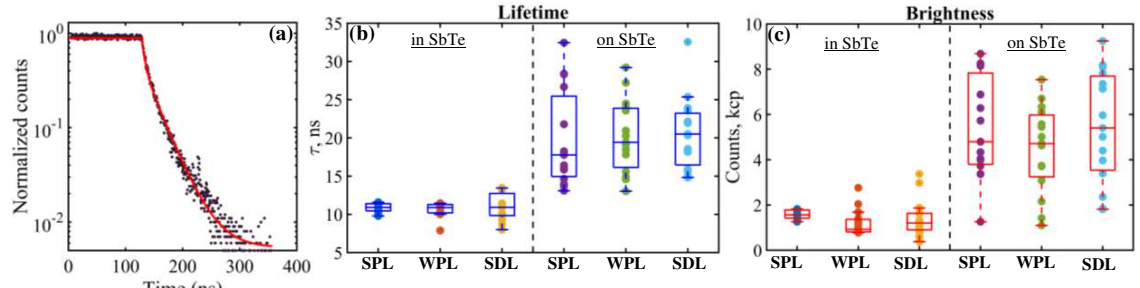

Time (ns)

Figure 2. (a) An example of time-correlated histogram of photon counts due to cathodoluminescence of nitrogenvacancy centers in a nanodiamond at the zero phonon line $\lambda=575 \mathrm{~nm}$. Black dots correspond to measured data, red line areas of SbTe films with SPL (strong plasmonic), WPL (wo of limes extracted from time-correlated histograms in marked in figures 1(b)-1(d). (c) The brightness of emission from nanodiamonds corresponding to the lifetime data in (b); kcp - kilocounts per second.

To understand the mechanism responsible for the observed changes in the lifetime statistics we modelled the emission of an isolated $120 \mathrm{~nm}$ large ND containing a single NV center with its electric dipole moment oriented parallel and orthogonal to the plane of the chalcogenide film. We considered two cases, as studied in our experiments, namely (i) an ND sitting on an SbTe film and (ii) an ND embedded in an SbTe film. Each case included films with WPL, SPL and SDL response, which were placed on a semi-infinite Si substrate. The modelling was performed in Comsol Multiphysics (see Supplementary Material for details). Figure 3 presents the simulation results obtained for a lossy SbTe film featuring SDL response in terms of the energy absorption rate in various parts of the structure and electric field emitted into vacuum by an $\mathrm{NV}$ center with its electric dipole moment oriented parallel to the substrate. Evidently, for an $\mathrm{ND}$ embedded in an SbTe film, the overall energy absorption rate is higher and the areas featuring high absorption are seen to extend further along with the film and into the Si substrate (compare figures 3(a) and 3(b)). Consequently, higher absorption should lead to stronger reduction of emission and that was exactly what we observed in our simulations (compare figures 3(c) and 3(d)). Similar differences in absorption and emission were observed for NDs on and in SbTe films featuring SPL and WPL response. 

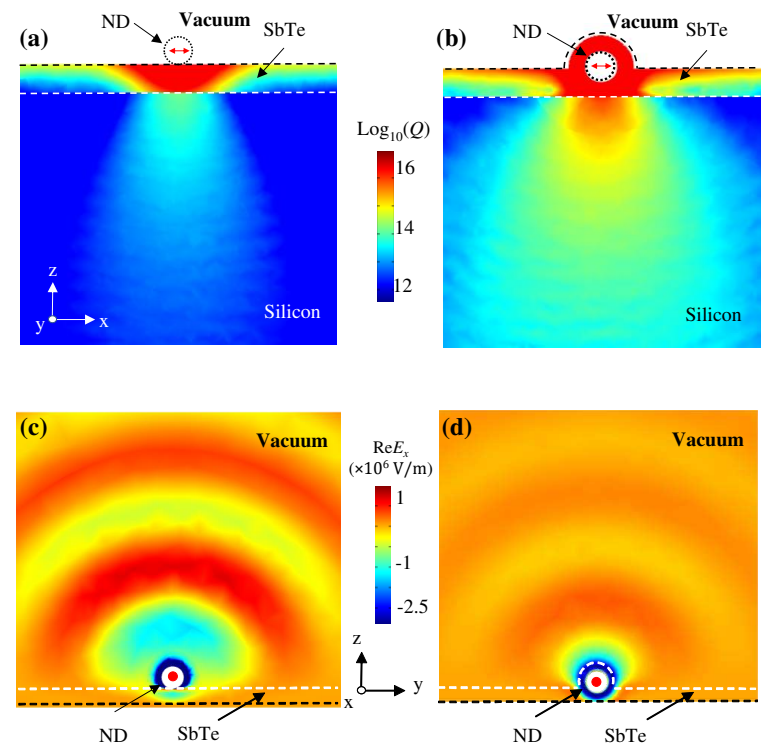

\begin{abstract}
Figure 3. (a) \& (b) Simulated distribution of absorption in the $x z$-plane presented in terms of rate of heat, $Q$, dissipated in the Si substrate and the SbTe film with a nanodiamond placed, respectively, on and in the film. The SbTe film here was modelled as a strong lossy dielectric (SDL) with $\varepsilon=7+i 20$. The electric dipole moment of a nitrogen-vacancy center inside the nanodiamond was oriented parallel to the $x$-axis, as shown by the red arrows. (c) \& (d) Simulated distribution of the $x$-component of the electric field (real part) in the $y z$-plane emitted into vacuum by the nitrogenvacancy center inside the nanodiamond placed, respectively, on and in SbTe film. The electric field is not plotted inside
\end{abstract} the nanodiamond.

The simulated data enabled us to calculate the radiative and non-radiative decay rates for all possible configurations (see Supplementary Material for details). The results are summarised and compared in Table S1. For the electric dipole moment of an NV center oriented perpendicular to the substrate, the non-radiative decay rate, $\gamma_{\text {non }}^{\perp}$, is about 4.5 times higher when an ND is embedded in as opposed to deposited on the SbTe film (for all three types of $\mathrm{SbTe}, \mathrm{SPL}, \mathrm{WPL}$, and WDL). Expectedly, the radiative decay rate, $\gamma_{\mathrm{rad}}^{\perp}$, in this case is lower - by a factor of 2 for plasmonic SbTe films and by a factor of 9 for a strongly dielectric SbTe film. When the electric dipole moment is parallel to the substrate the situation is similar, though the differences in the decay rates, $\gamma_{\text {non }}^{\|}$and $\gamma_{\text {rad }}^{\|}$, appear to be larger: $\gamma_{\text {non }}^{\|}$is $>18$ times higher and $\gamma_{\mathrm{rad}}^{\|}$is $>3$ times lower for all three types of an SbTe film incorporating an ND. We further note that the ratio between the non-radiative and radiative decay rates does not exceed 2 for an ND sitting on an SbTe film, but raises above 9 for an ND embedded in an SbTe film (approaching 174 when SbTe behaves as a strong dielectric and the electric dipole moment is parallel to the substrate). Our analysis, therefore, indicates that the experimentally observed narrowing of the lifetime distribution was enforced by substantially increased non-radiative decay, which completely dominates the relaxation of NV centers in embedded NDs. The enhancement of non-radiative decay should lead to shortening of the average lifetime in accordance with our experimental observations (see figure 2(b)).

The calculated data in table S2 also suggests that there is another mechanism responsible for 
changes in the lifetime statistics - an interplay between scattering and absorption of emitted photons. For an ND embedded in an SbTe film, it renders the total decay rate, $\gamma_{\text {tot }}$, of an NV center almost insensitive to the orientation of its electric dipole moment $\left(\gamma_{\text {tot }}^{\perp} / \gamma_{\text {tot }}^{\|} \sim 1\right)$. Thus, if such an ND contains many NV centers (as our samples) this mechanism will efficiently negate the variation of lifetimes across an ND due to random orientations of the electric dipole moments. ${ }^{12}$ On the contrary, for an ND sitting on an SbTe film the dependence of $\gamma_{\text {tot }}$ on the electric dipole alignment remains quite strong $\left(\gamma_{\text {tot }}^{\perp} / \gamma_{\text {tot }}^{\|}>3.5\right)$ and, therefore, can only lead to further spreading of the lifetime distribution (especially when SbTe exhibits plasmonic response).

In conclusion, we have experimentally demonstrated that embedding nanodiamonds with multiple nitrogen-vacancy centers in thin chalcogenide films enabled us to dramatically narrow the distribution of the decay rates and shorten the average lifetime of the fluorescent defects. Our numerical analysis has shown that the observed changes in the lifetime statistics, i.e., more than five-fold reduction of the spread and two-fold decrease of the average, were underpinned by two mechanisms. One of the mechanisms is dissipation in lossy chalcogenide films, which enhances non-radiative decay to the extent that it completely dominates radiative decay for nanodiamonds embedded in the films. While such a mechanism naturally reduces the brightness of a single nitrogen-vacancy center, in practice the intensity of fluorescence (and, in our case, cathodoluminescence) can be maintained at a sufficient level in nanodiamonds containing many centers. The other mechanism corresponds to an interplay between scattering and absorption of emitted photons. For embedded nanodiamonds it renders the total decay rate of nitrogen-vacancy centers insensitive to the orientation of their electric dipole moments, which is random in large ensembles of the defects. Given that one can dial (via stoichiometric engineering) optical response of chalcogenides anywhere from strongly plasmonic to strongly dielectric, ${ }^{35}$ and also can vary the level of losses by switching (optically, electrically or thermally) between amorphous and crystalline phases, ${ }^{39-41}$ our approach offers a straightforward way of implementing active control over the emission statistics in nanodiamonds.

\section{Supplementary material}

See supplementary material for CL spectrum of NV centres in nanodiamond in SbTe film, carrier lifetime distributions and the calculating lifetimes of an NV centre in a nanodiamond.

\section{Acknowledgements}

The authors would like to acknowledge the financial support of the UK's Engineering and Physical Sciences Research Council (Grants No. EP/M0091221), Nanyang Technological University Strategic Research Facilities Initiative and Singapore ASTAR QTE program (No. SERC A1685b0005), China Scholarship Council (No. 201608440362).

\section{Data availability}

Following a period of embargo, the data from this paper will be available from the University of Southampton ePrints research repository: http://doi.org/10.5258/SOTON/D1825. 
L., The nitrogen-vacancy colour centre in diamond. Physics Reports 2013, 528 (1), 1-45.

2. Gruber, A.; Dräbenstedt, A.; Tietz, C.; Fleury, L.; Wrachtrup, J.; Von Borczyskowski, C., Scanning confocal optical microscopy and magnetic resonance on single defect centers. Science 1997, 276 (5321), 2012-2014.

3. C. Kurtsiefer, S. M., P. Zarda, and H. Weinfurter, Stable Solid-State Source of Single Photons. Phys Rev Lett 2000, 85 (2).

4. R. Brouri, A. B., J.-. Poizat, and P. Grangier, Photon antibunching in the fluorescence of individual color centers in diamond. Optics Letters 2000, 25 (17).

5. Beveratos, A.; Brouri, R.; Gacoin, T.; Poizat, J.-P.; Grangier, P., Nonclassical radiation from diamond nanocrystals. Physical Review A 2001, 64 (6).

6. Storteboom, J.; Dolan, P.; Castelletto, S.; Li, X.; Gu, M., Lifetime investigation of single nitrogen vacancy centres in nanodiamonds. Opt Express 2015, 23 (9), 11327-33.

7. Robins, L. H.; Cook, L. P.; Farabaugh, E. N.; Feldman, A., Cathodoluminescence of defects in diamond films and particles grown by hot-filament chemical-vapor deposition. Physical Review $B$ 1989, 39 (18), 13367-13377.

8. Tallaire, A.; Collins, A. T.; Charles, D.; Achard, J.; Sussmann, R.; Gicquel, A.; Newton, M. E.; Edmonds, A. M.; Cruddace, R. J., Characterisation of high-quality thick single-crystal diamond grown by CVD with a low nitrogen addition. Diamond and Related Materials 2006, 15 (10), 1700-1707.

9. Tizei, L. H.; Kociak, M., Spectrally and spatially resolved cathodoluminescence of nanodiamonds: local variations of the NV(0) emission properties. Nanotechnology 2012, 23 (17), 175702.

10. Tizei, L. H.; Kociak, M., Spatially resolved quantum nano-optics of single photons using an electron microscope. Phys Rev Lett 2013, 110 (15), 153604.

11. Meuret, S.; Tizei, L. H.; Cazimajou, T.; Bourrellier, R.; Chang, H. C.; Treussart, F.; Kociak, M., Photon bunching in cathodoluminescence. Phys Rev Lett 2015, 114 (19), 197401.

12. Lourenço-Martins, H.; Kociak, M.; Meuret, S.; Treussart, F.; Lee, Y. H.; Ling, X. Y.; Chang, H.C.; Galvão Tizei, L. H., Probing Plasmon-NVO Coupling at the Nanometer Scale with Photons and Fast Electrons. ACS Photonics 2017, 5 (2), 324-328.

13. Zhang, H.; Glenn, D. R.; Schalek, R.; Lichtman, J. W.; Walsworth, R. L., Efficiency of Cathodoluminescence Emission by Nitrogen-Vacancy Color Centers in Nanodiamonds. Small 2017, 13 (22).

14. Feldman, M. A.; Dumitrescu, E. F.; Bridges, D.; Chisholm, M. F.; Davidson, R. B.; Evans, P. G.; Hachtel, J. A.; Hu, A.; Pooser, R. C.; Haglund, R. F.; Lawrie, B. J., Colossal photon bunching in quasiparticle-mediated nanodiamond cathodoluminescence. Physical Review B 2018, 97 (8).

15. Gur, F. N.; McPolin, C. P. T.; Raza, S.; Mayer, M.; Roth, D. J.; Steiner, A. M.; Loffler, M.; Fery, A.; Brongersma, M. L.; Zayats, A. V.; Konig, T. A. F.; Schmidt, T. L., DNA-Assembled Plasmonic Waveguides for Nanoscale Light Propagation to a Fluorescent Nanodiamond. Nano Lett 2018, 18 (11), 7323-7329.

16. Malykhin, S. A.; Houard, J.; Ismagilov, R. R.; Orekhov, A. S.; Vella, A.; Obraztsov, A. N., Luminescent Characteristics of Needle-Like Single Crystal Diamonds. physica status solidi (b) 2018, 255 (1).

17. Lim, T. S.; Fu, C. C.; Lee, K. C.; Lee, H. Y.; Chen, K.; Cheng, W. F.; Pai, W. W.; Chang, H. C.; Fann, W., Fluorescence enhancement and lifetime modification of single nanodiamonds near a nanocrystalline silver surface. Phys Chem Chem Phys 2009, 11 (10), 1508-14.

18. S. Schietinger, M. B., T. Aichele, and O. Benson, Plasmon-Enhanced Single Photon Emission from a Nanoassembled Metal-Diamond Hybrid Structure at Room Temperature. Nano Lett 2009, 9 (4), 1694-1698.

19. Chi, Y.; Chen, G.; Jelezko, F.; Wu, E.; Zeng, H., Enhanced Photoluminescence of Single-Photon Emitters in Nanodiamonds on a Gold Film. IEEE Photonics Technology Letters 2011, 23 (6), 374-376.

20. Choy, J. T.; Hausmann, B. J. M.; Babinec, T. M.; Bulu, I.; Khan, M.; Maletinsky, P.; Yacoby, A.; Lončar, M., Enhanced single-photon emission from a diamond-silver aperture. Nature Photonics 
2011, 5 (12), 738-743.

21. Chang, H.-C.; Hsiao, W. W.-W.; Su, M.-C., Fluorescent nanodiamonds. John Wiley \& Sons: 2018.

22. Lenzini, F.; Gruhler, N.; Walter, N.; Pernice, W. H. P., Diamond as a Platform for Integrated Quantum Photonics. Advanced Quantum Technologies 2018, 1 (3).

23. Bradac, C.; Gao, W.; Forneris, J.; Trusheim, M. E.; Aharonovich, I., Quantum nanophotonics with group IV defects in diamond. Nat Commun 2019, 10 (1), 5625.

24. Radulaski, M.; Zhang, J. L.; Tzeng, Y. K.; Lagoudakis, K. G.; Ishiwata, H.; Dory, C.; Fischer, K. A.; Kelaita, Y. A.; Sun, S.; Maurer, P. C.; Alassaad, K.; Ferro, G.; Shen, Z. X.; Melosh, N. A.; Chu, S.; Vučković, J., Nanodiamond Integration with Photonic Devices. Laser \& Photonics Reviews 2019, 13 (8). 25. Castelletto, S.; Rosa, L.; Boretti, A., Micro-manipulation of nanodiamonds containing NV centers for quantum applications. Diamond and Related Materials 2020, 106.

26. Lee, J.; Leong, V.; Kalashnikov, D.; Dai, J.; Gandhi, A.; Krivitsky, L., Hybrid Quantum Photonics. arXiv preprint arXiv:2005.11001 2020.

27. Schell, A. W.; Engel, P.; Werra, J. F.; Wolff, C.; Busch, K.; Benson, O., 16-Scanning single quantum emitter fluorescence lifetime imaging: quantitative analysis of the local density of photonic states. Nano Lett 2014, 14 (5), 2623-7.

28. Schirhagl, R.; Chang, K.; Loretz, M.; Degen, C. L., Nitrogen-vacancy centers in diamond: nanoscale sensors for physics and biology. Annu Rev Phys Chem 2014, 65, 83-105.

29. Mohtashami, A.; Femius Koenderink, A., Suitability of nanodiamond nitrogen-vacancy centers for spontaneous emission control experiments. New Journal of Physics 2013, 15 (4).

30. Reineck, P.; Trindade, L. F.; Havlik, J.; Stursa, J.; Heffernan, A.; Elbourne, A.; Orth, A.; Capelli, M.; Cigler, P.; Simpson, D. A.; Gibson, B. C., Not All Fluorescent Nanodiamonds Are Created Equal: A Comparative Study. Particle \& Particle Systems Characterization 2019, 36 (3).

31. Shilkin, D. A.; Shcherbakov, M. R.; Lyubin, E. V.; Katamadze, K. G.; Kudryavtsev, O. S.; Sedov, V. S.; Vlasov, I. I.; Fedyanin, A. A., Optical magnetism and fundamental modes of nanodiamonds. ACS Photonics 2017, 4 (5), 1153-1158.

32. Zalogina, A. S.; Savelev, R. S.; Ushakova, E. V.; Zograf, G. P.; Komissarenko, F. E.; Milichko, V. A.; Makarov, S. V.; Zuev, D. A.; Shadrivov, I. V., Purcell effect in active diamond nanoantennas. Nanoscale 2018, 10 (18), 8721-8727.

33. Lukosz, W.; Kunz, R., Fluorescence lifetime of magnetic and electric dipoles near a dielectric interface. Optics Communications 1977, 20 (2), 195-199.

34. Schniepp, H.; Sandoghdar, V., Spontaneous emission of europium ions embedded in dielectric nanospheres. Physical review letters 2002, 89 (25), 257403.

35. Piccinotti, D.; Gholipour, B.; Yao, J.; MacDonald, K. F.; Hayden, B. E.; Zheludev, N. I., Stoichiometric Engineering of Chalcogenide Semiconductor Alloys for Nanophotonic Applications. Adv Mater 2019, 31 (14), e1807083.

36. Guerin, S.; Hayden, B.; Hewak, D. W.; Vian, C., Synthesis and Screening of Phase Change Chalcogenide Thin Film Materials for Data Storage. ACS Comb Sci 2017, 19 (7), 478-491.

37. Hayden, S. G. a. B. E., Physical Vapor Deposition Method for the High-Throughput Synthesis of Solid-State Material Libraries. J. Comb. Chem 2006, 8 (66).

38. Sola-Garcia, M.; Meuret, S.; Coenen, T.; Polman, A., Electron-Induced State Conversion in Diamond NV Centers Measured with Pump-Probe Cathodoluminescence Spectroscopy. ACS Photonics 2020, 7 (1), 232-240.

39. Karvounis, A.; Gholipour, B.; MacDonald, K. F.; Zheludev, N. I., All-dielectric phase-change reconfigurable metasurface. Applied Physics Letters 2016, 109 (5), 051103.

40. Gholipour, B.; Karvounis, A.; Yin, J.; Soci, C.; MacDonald, K. F.; Zheludev, N. I., Phase-changedriven dielectric-plasmonic transitions in chalcogenide metasurfaces. NPG Asia Materials 2018, 10 (6), 533-539.

41. Gholipour, B.; Piccinotti, D.; Karvounis, A.; MacDonald, K. F.; Zheludev, N. I., Reconfigurable ultraviolet and high-energy visible dielectric metamaterials. Nano letters 2019, 19 (3), 1643-1648. 


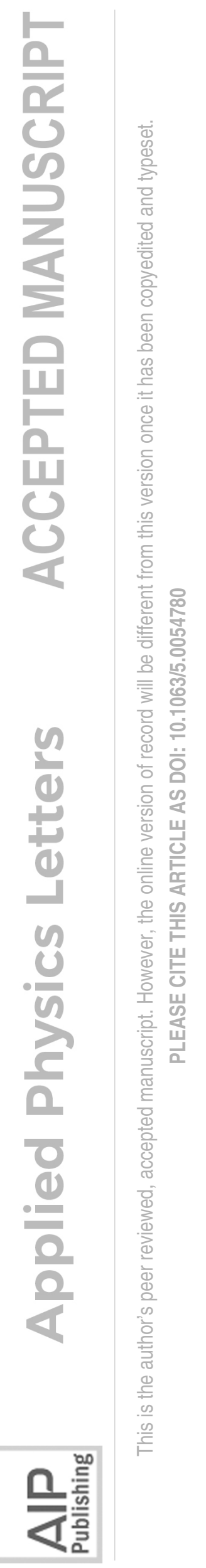




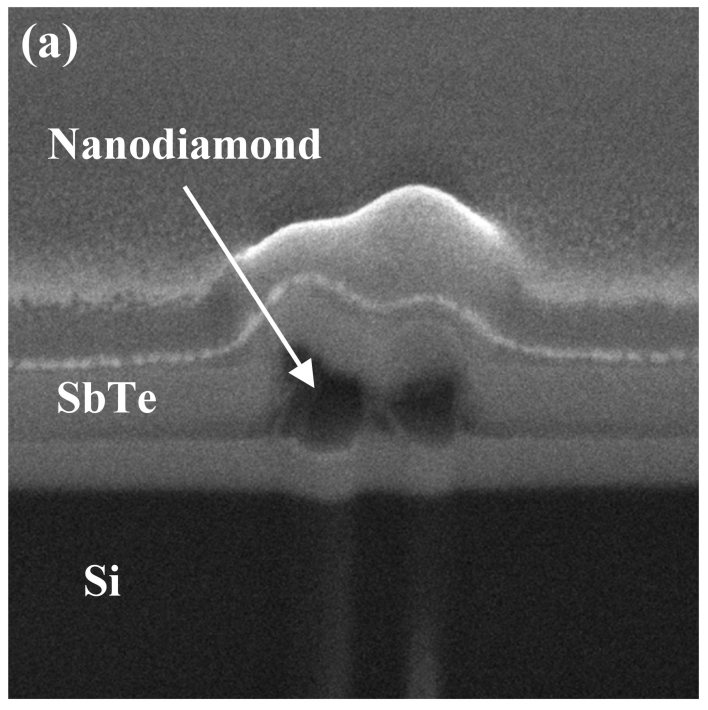

(b) $\operatorname{Re} \varepsilon$ of SbTe film at $\lambda=575 \mathrm{~nm}$

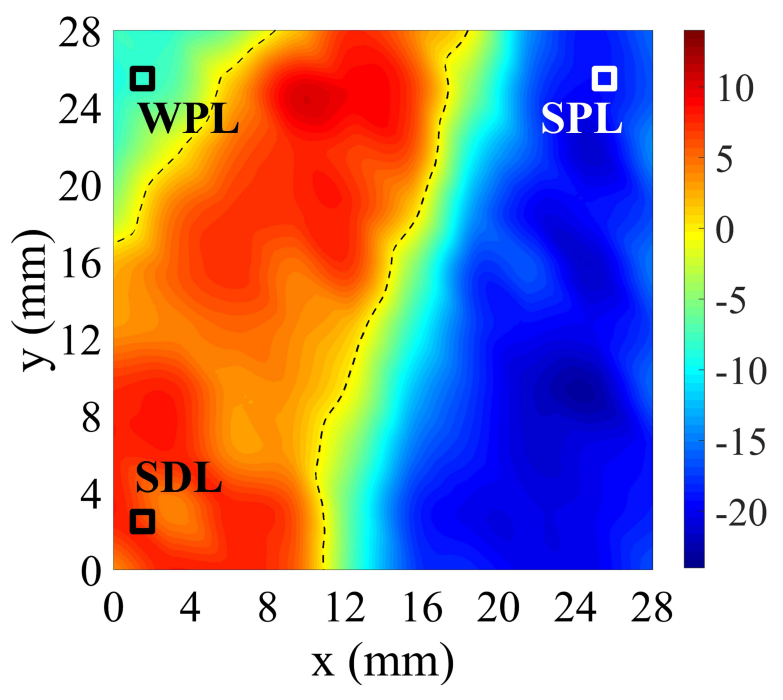

(c) $\operatorname{Im} \varepsilon$ of SbTe film at $\lambda=575 \mathrm{~nm}$

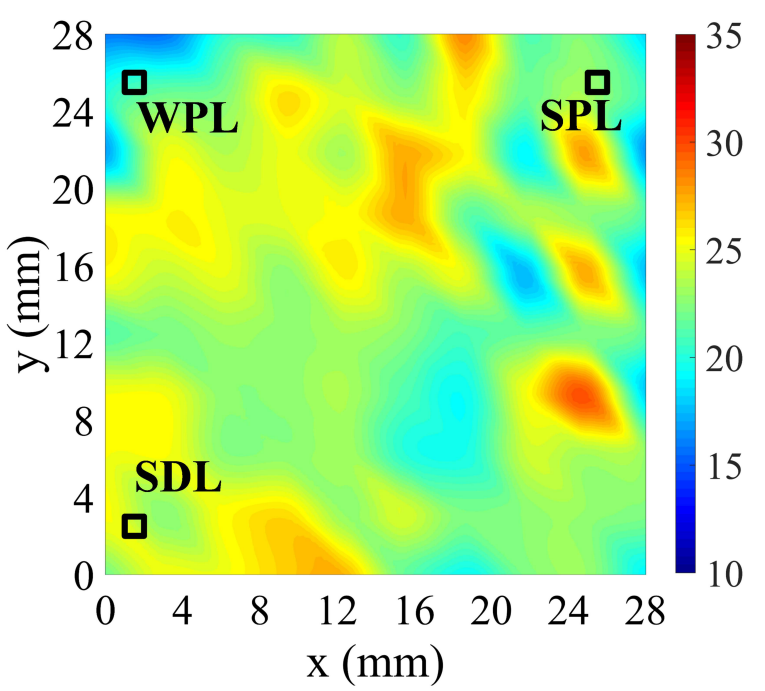

(d) Thickness of SbTe film (nm)

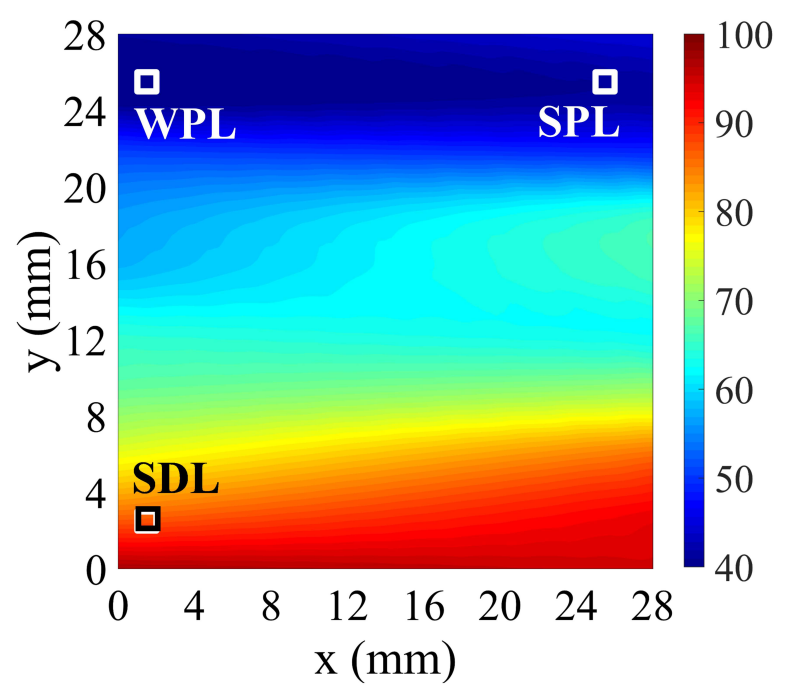




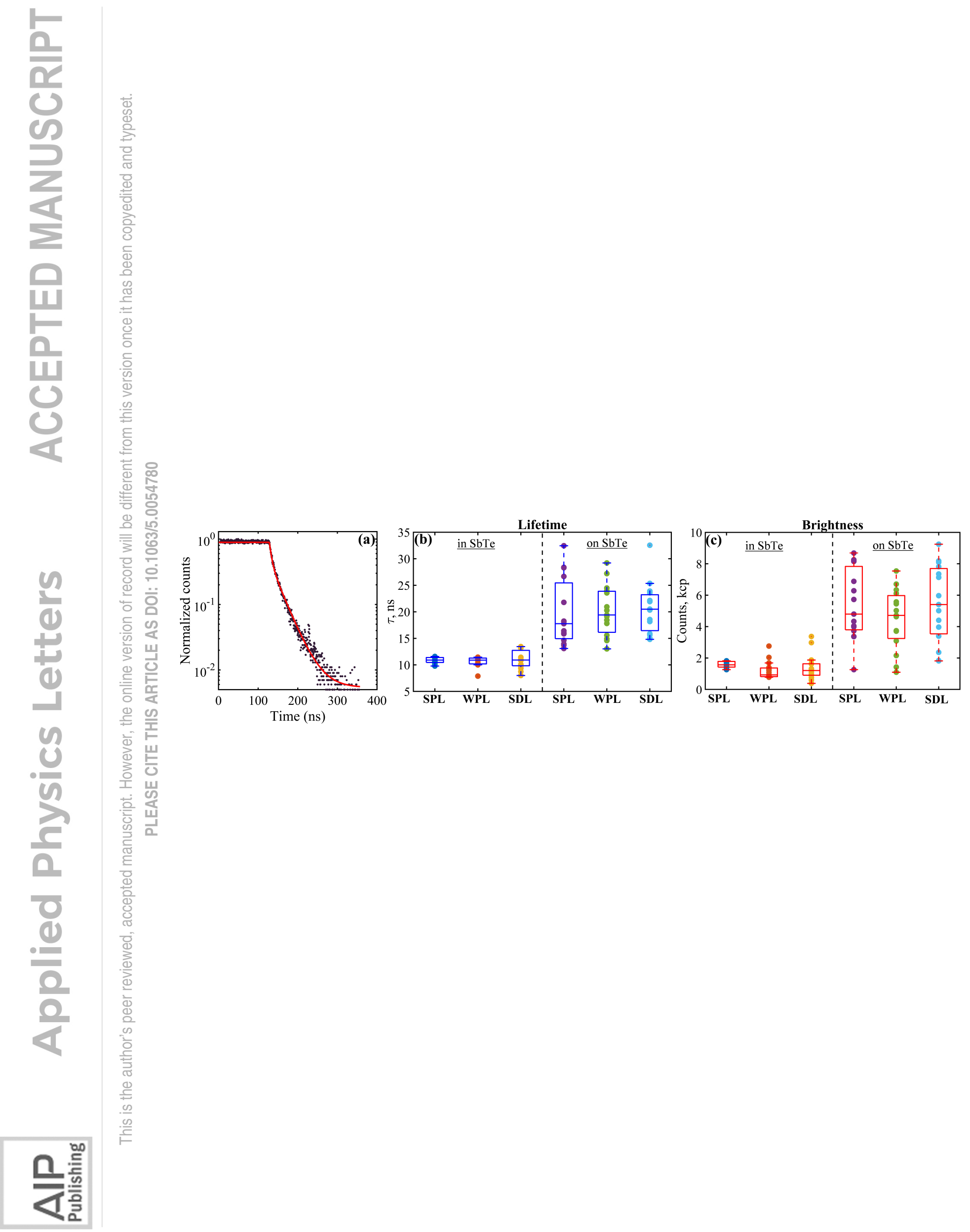



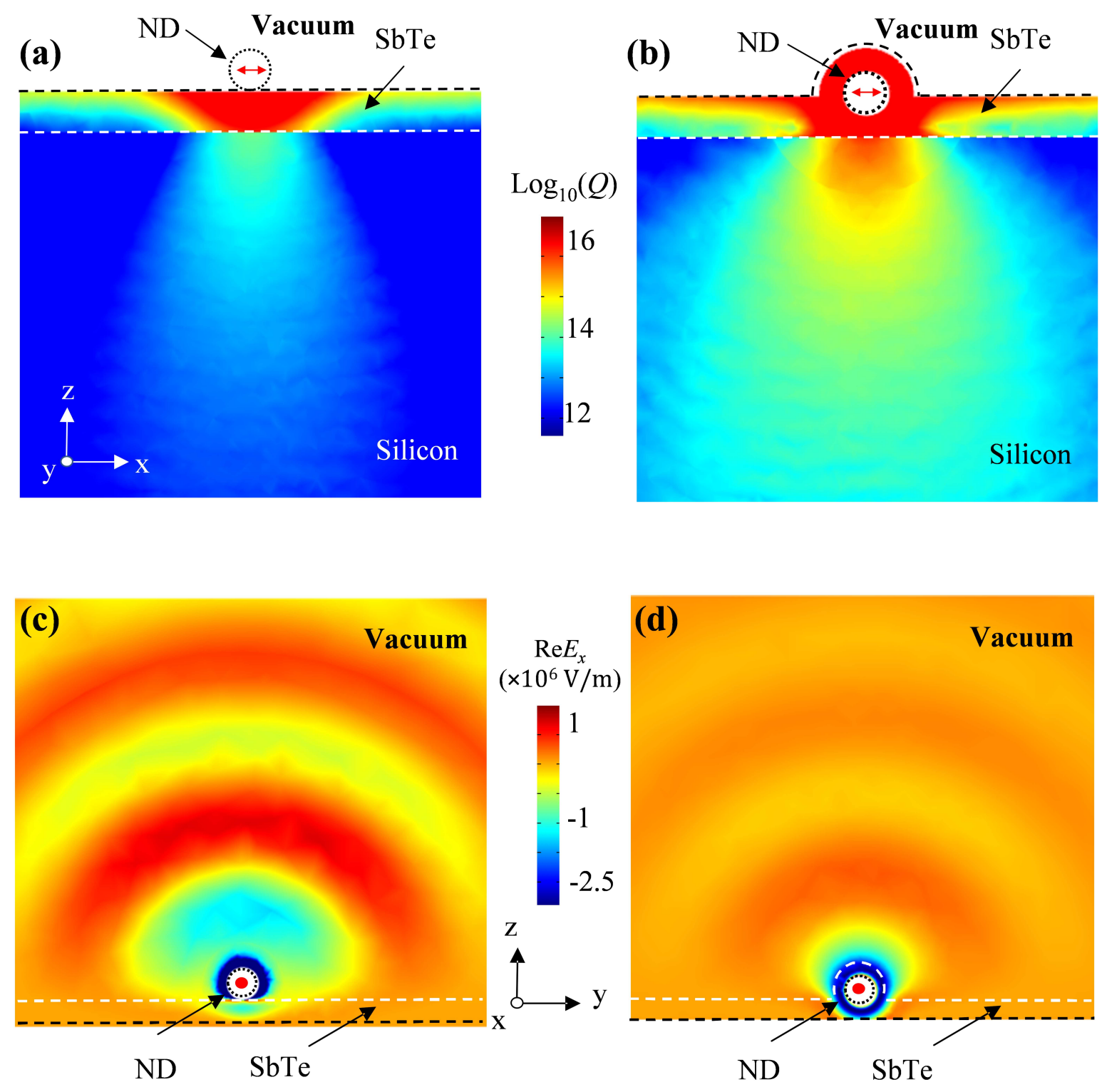